Introduction Evidence suggests that sex partner meeting places may be important locales to access men who have sex with men (MSM) to implement targeted HIV control strategies. Little is known about how patterns of these places have changed over time and whether there are new and emerging meeting places.

Methods The objectives of this study were to 1) describe the frequency of report of MSM sex partner meeting places over time, 2) to compare frequently reported meeting places over a five year period and in the past year, and 3) in a subset analysis, compare distributions of mean HIV viral loads, as a proxy for HIV transmission risk, of reported meeting places among newly HIV diagnosed MSM in Baltimore City, Maryland.

Results 869 sex partner meeting places were reported, including 306 unique places. Internet-based sites (38\%) and bars/clubs (31\%) were the most frequently reported meeting place typologies. Over the five year period there was variability in the frequencies of reports of meeting place typologies over time. Among internet-based sites and bars/clubs, 3/5 and 4/5 respectively most frequently reported sites over the five years were also in the top five most frequently reported in the most recent year. The distribution of mean viral load by place typology was not significantly different overall (Fisher's exact $p=0.700$ ) or between bars/clubs compared to internet-based sites (Fisher's exact $\mathrm{p}=0.863$ ).

Conclusion This study provides a richer understanding of sex partner meeting places reported by MSM over time and their transmission potential.

Disclosure of interest statement The Centre for Child and Community Health Research (CCHR) and the Baltimore City Health Department were funded for this work by the Centres for Disease Control and Prevention and the NIH. No pharmaceutical grants were received in the development of this study.

\section{P04.23 MIND THE GAP! BIOLOGICAL AND BEHAVIOURAL CONCURRENCY AND THEIR POTENTIAL FOR STI TRANSMISSION IN THE BRITISH POPULATION}

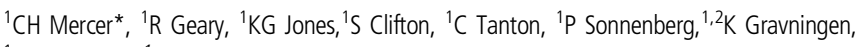
${ }^{1}$ AM Johnson. ${ }^{1}$ Centre for Sexual Health \& HIV Research, Research Department of Infection \& Population Health, University College London; ${ }^{2}$ Department of Microbiology and Infection
\end{abstract} Control, University Hospital of North Norway

\subsection{6/sextrans-2015-052270.277}

Background Key drivers for STI transmission are the number of sexual partners and their timing. Concurrent partnerships are a known risk factor but non-overlapping (serially monogamous) partnerships may be biologically concurrent for some STIs where infectious periods extend across the 'gap'. We examine recent partnership histories among people aged 16-44y in Britain and how they relate to reported STI diagnoses.

Methods Analyses of data collected for Britain's third National Survey of Sexual Attitudes and Lifestyles, a probability survey undertaken 2010-2012 with 9,902 people aged 16-44y. Computer-assisted self-interviews included questions about participants' 3 most recent partners and STI diagnosis history. Gaps between partners were calculated from the dates of most recent sex with a former partner and first sex with the next partner.

Results Over $90 \%$ of men and women reported $>=1$ partners in the past $5 \mathrm{y}$, of whom, $51.2 \%$ (men) and $42.5 \%$ (women) reported $>=2$ partners during this time and so had had $>=1$ gaps (total: 7, 724 gaps). The median gap was 2 months (IQR:-3 to 8 months) with two-thirds of all gaps $>=1$ month denoting serial monogamy. One-quarter (95\% CI: $23.8 \%-26.2 \%$ ) of all gaps were 0-3 months, while $21.8 \%$ (95\% CI: 20.7\%-22.9\%) were $>=12$ months. In multivariable analyses which adjusted for total partner numbers (past 5y), reported STI diagnoses (past $5 y$ ) were as common among participants whose recent partners had been concurrent as serially monogamous participants where the gap was $<=4$ months among men: $10.3 \%$ (95\% CI: 7.6\%-13.9\%) vs. $11.3 \%$ (95\% CI: $8.9 \%-14.3 \%)$, and $<=12$ months among women: $12.3 \%$ (95\% CI: 9.2\%-16.3\%) vs. 11.5\% (95\% CI: 9.7\%-13.6\%).

Conclusion While people in Britain have few partners on average and the majority with multiple partners are serially monogamous, the gap between partners is often shorter than the infectious period of common STIs and so effectively biologically concurrent. STI prevention efforts need to address this and should encourage STI testing prior to unprotected sex with new partners, even if serially monogamous.

Disclosure of interest statement AMJ has been a Governor of the Wellcome Trust since 2011. The other authors declare that they have no conflicts of interest.

\section{P04.24 EVALUATION OF PREVALENCE OF STIS BETWEEN WOMEN REFERRED TO "COUNSELLING AND HARM REDUCTION CENTRE FOR VULNERABLE WOMEN" IN ISFAHAN - IRAN 2014}

M Meshkati*, S Narimani, M Zandieh, R Fadai Nobari. Isfahan University of Medical Sceiences, Province Health Center

\subsection{6/sextrans-2015-052270.278}

Introduction At this time sexually transmitted infections are a major public health problem. Prevalence of STIs is correlated with distribution of sexual behaviours. Rates of sexual activity among Iranian youth were low but it is gradually changing. In the other hand rate of sexual transmission of HIV among newly identified people living with HIV is increasing year by year that might have probably a consistent trend with STIs. By this way Counselling and Harm Reduction centres for vulnerable women were established. So we looked clients in these centres for evaluating prevalence of STIs base on syndromic approach.

Methods In Isfahan, we lunched 2 centres for vulnerable women. We performed a survey (cross - sectional) for all clients who referred in 2014. The data was gathered by the client's files.

Results We have admitted 160 clients, the majority of them were in group ages $25-34$ years (38.4\%) and married (81\%). $47 \%$ of clients had history of sexual high risk behaviour that $50 \%$ were drug abuser. All of clients were assessed in every visit to centre for signs of STIs.30\% of them need to exam and 50\% of them were treated because vaginal discharge.

Conclusion These results show the increase of sexual high risk behaviours in young people and special between drug abuser. So it is necessary to evaluate STIs between this group. In this time, we have to manage base on syndromic approach so we need to provide etiologic approach for improving care and treatment. We have faced risk of third episode of HIV/AIDS from IDU to sexual contact in Iran right now so it is essential to improve harm reduction centres as well as social support in order to empower women for job finding that could play an effective and important role in control of spreading HIV/AIDS and STIs.

Disclosure of interest Counselling and Harm Reduction centres for vulnerable women were founded by Isfahan university of medical sciences. 